\title{
Recent results on Heavy Flavor production with the CMS experiment
}

\author{
Vincenzo Mastrapasqua ${ }^{a, \dagger, *}$ \\ a Dipartimento Interateneo di Fisica, Università degli Studi di Bari "Aldo Moro", \\ via Giovanni Amendola n.173, 70126 - Bari, Italy \\ E-mail: vincenzo.mastrapasqua@cern.ch
}

The present report summarizes three recent CMS results on Heavy Flavor production, fully or partially exploiting the data collected at LHC with proton-proton collisions at the center-of-mass energy $\sqrt{s}=13 \mathrm{TeV}$. Firstly, a measurement of the prompt open-charm differential production cross section is performed on $29 \mathrm{nb}^{-1}$ of data collected in 2016. The CMS results are compared to a few theoretical calculations and to previous measurements by other experiments at the LHC in different kinematic ranges. Secondly, the observation of two $B_{c}$ excited states by CMS, $B_{c}(2 S)^{+}$and $B_{c}^{*}(2 S)^{+}$, has been recently followed by the measurement of their relative production cross sections using $143 \mathrm{fb}^{-1}$ of data collected during the whole LHC Run-II (2015-2018). The dependence of the cross sections on the transverse momentum and the rapidity of the ground state $B_{c}$ is studied along with the dipion invariant mass distribution. Finally, a fiducial cross-section for $\Upsilon(1 S)$ pair production is measured using $35.9 \mathrm{fb}^{-1}$ of data collected during 2016. The contribution of double-parton scattering (DPS) to the total inclusive $\Upsilon(1 S)$ pair production cross section is also measured for the first time in the same fiducial region.

BEAUTY2020

21-24 September 2020

Kashiwa, Japan (online)
*Speaker
${ }^{\dagger}$ On behalf of the CMS Collaboration 


\section{Measurement of prompt open-charm differential production cross sections}

The following measurement on prompt open-charm production is the first result on charm differential production cross sections carried out by the CMS experiment [1], in proton-proton (pp) collisions at the center-of-mass energy $\sqrt{s}=13 \mathrm{TeV}$ [2]. It is performed on a small integrated luminosity sample $\left(29 \mathrm{nb}^{-1}\right)$ collected in 2016. Data are selected with the most inclusive - but heavily prescaled - available trigger (ZeroBias) that requires only the presence of colliding beams.

The search aims to reconstruct promptly-produced D mesons, namely either coming from the pp collision point (primary vertex, $\mathrm{PV}$ ) or from other excited charm resonances produced promptly. The focus is on the following exclusive channels: $\mathrm{pp} \rightarrow D^{*+} \mathrm{X} \rightarrow D^{0} \pi_{\text {slow }}^{+} \mathrm{X} \rightarrow K^{-} \pi^{+} \pi_{\text {slow }}^{+} \mathrm{X}$, $\mathrm{pp} \rightarrow D^{0} \mathrm{X} \rightarrow K^{-} \pi^{+} \mathrm{X}$ and $\mathrm{pp} \rightarrow D^{+} \mathrm{X} \rightarrow K^{-} \pi^{+} \pi^{+} \mathrm{X}$ (charge-conjugation is implied). The main possible source of contamination consists of charmed hadrons coming from beauty hadrons. A wide kinematic region in transverse momentum $\left(p_{T}\right)$ and pseudo-rapidity $(\eta)$ of the $D$ mesons is explored: $4<p_{T}(D)<100 \mathrm{GeV}$ and $|\eta(D)|<2.1$.

Two (three) charged tracks are combined to form a $D^{0}\left(D^{+}\right)$candidate, which is defined by the total charge and an invariant mass window specific for each meson. Since CMS lacks hadronic particle identification, a kaon or pion mass hypothesis is assumed for each track according to the charge of the candidate and the specific decay channel. Further cuts require the cosine of the angle between the charm candidate momentum and the vector pointing from the PV to the secondary vertex (SV) to be greater than 0.99 and the significance of the decay length to be greater than a value specific for each meson (>3, 5, 10 for $D^{*+}, D^{0}, D^{+}$respectively). The $D^{*+}$ candidate reconstruction is completed by adding a third track, corresponding to the slow pion, with looser selection and that must be kinematically combined with the $D^{0}$ candidate. The arbitration criterion for candidates' multiplicity is to keep the candidate with the invariant mass value closest to the nominal one for $D^{+}$ and $D^{0}$ and the candidate with the lowest $\Delta \mathrm{M}=\mathrm{M}\left(\mathrm{K} \pi \pi_{\text {slow }}\right)-\mathrm{M}(\mathrm{K} \pi)$ for $D^{*+}$.

The prompt open-charm differential production cross section $d \sigma / d p_{T}$ is measured in $9 p_{T}$ bins in the chosen kinematic range. The signal yields are determined with an unbinned maximumlikelihood (UML) fit to the invariant mass distributions ( $\Delta \mathrm{M}$ for $D^{*+}$ ) in each $p_{T}$ bin independently. The signal model is the sum of two gaussian functions with common mean but different variance, in order to describe the non-uniform resolution over the detector acceptance, whereas the combinatorial background is modeled with different functions according to the decay mode.

The non-prompt contamination contribution is evaluated with Monte Carlo (MC) simulations and then bin-by-bin subtracted from the visible yield. This contribution is not negligible and ranges from $5 \%$ to $17 \%$. MC simulations are also used to estimate bin-by-bin the overall reconstruction efficiency and acceptance in the kinematic range and evaluate systematic uncertainties.

The differential $D$ meson prompt-production cross-section in $p_{T}$ bins can be expressed as:

$$
\frac{d \sigma(p p \rightarrow D X)}{d p_{T}}=\frac{N_{i}(D \rightarrow f)}{\Delta p_{T} \mathcal{B}(D \rightarrow f) \mathcal{L} \varepsilon_{i, t o t}(D \rightarrow f)}
$$

where $N_{i}(D \rightarrow f)$ is the number of prompt candidates in the selected final state $f$ for the $i$-th bin, $\Delta p_{T}$ is the bin width, $\mathcal{B}(D \rightarrow f)$ is the branching fraction of the reconstructed final state, $\varepsilon_{i, t o t}(D \rightarrow f)$ is the total reconstruction efficiency of the decay chain evaluated in the simulated event sample, and $\mathcal{L}$ is the considered integrated luminosity. Data are compared with a few simulation models and theoretical predictions (Fig. 1). 

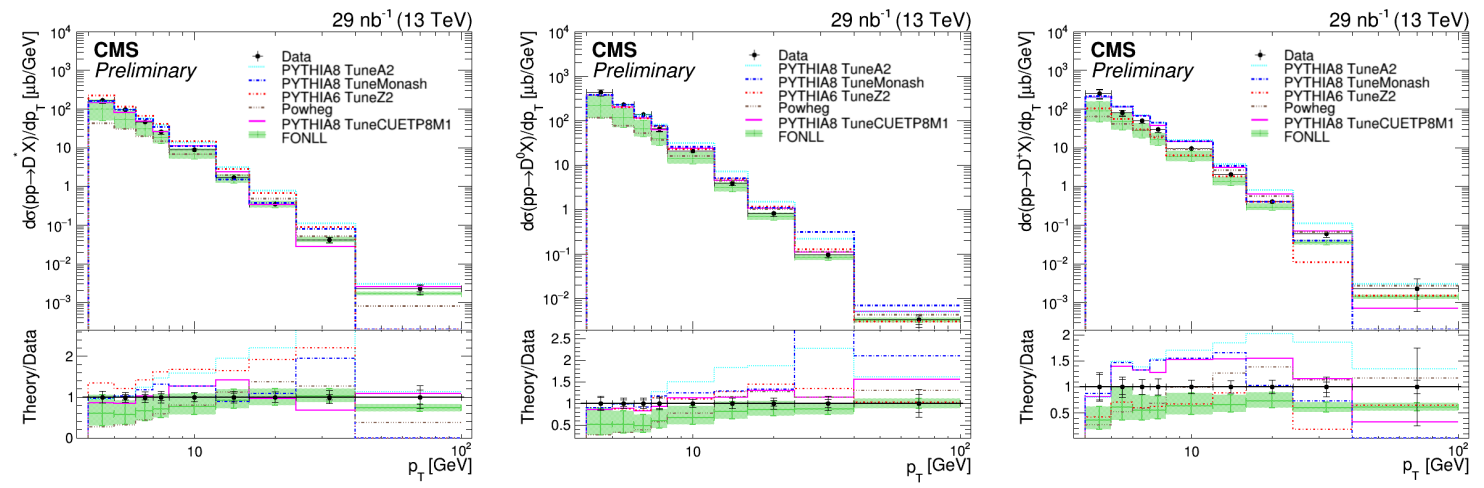

Figure 1: Differential prompt production cross section as a function of $p_{T}$ for $D^{*+}($ left $), D^{0}$ (center) and $D^{+}($right $)$. The considered theoretical models are in good agreement with data (black points). The error bars are the root sum squared of statistical and systematical uncertainties. The ratio of theoretical prediction over data is shown in the lower panels. [2]

The FONLL calculations [3] provide the most stable description of data and are used to compare the new CMS results with previous measurements obtained by other LHC collaborations. Here only a representative selection of comparisons is shown, in order to describe the evolution in the center-of-mass energy and the kinematic dependencies. Since CMS and ATLAS detectors have the same geometrical acceptance, the scaling of the cross section with the center-of-mass energy can be observed by comparing CMS data at $\sqrt{s}=13 \mathrm{TeV}$ with ATLAS results obtained at $\sqrt{s}=7 \mathrm{TeV}$ [4] (Fig. 2 left). The second comparison is between CMS and LHCb data, both collected at $\sqrt{s}=13 \mathrm{TeV}$ [5] (Fig. 2 right). The complementary phase-space acceptances of the CMS and LHCb detectors can extend the investigation on charm production cross sections over a wide kinematic range. All sets of measurements are in good agreement with the corresponding FONLL predictions.
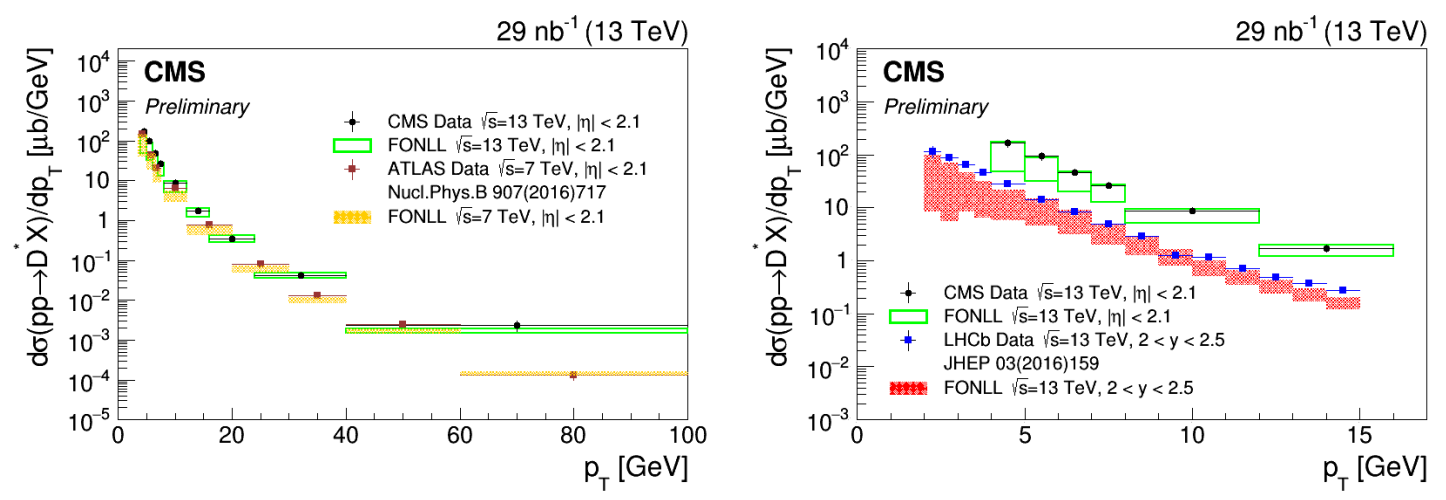

Figure 2: CMS data for $D^{*}$ differential prompt production cross section at $\sqrt{s}=13 \mathrm{TeV}$ [2] are compared with ATLAS results at $\sqrt{s}=7 \mathrm{TeV}$ [4] (left) and LHCb results at $\sqrt{s}=13 \mathrm{TeV}$ [5] (right). A meaningful comparison with $\mathrm{LHCb}$ is obtained by showing only CMS data with $p_{T}<16 \mathrm{GeV}$ and LHCb data with $2<y<2.5$. All sets of measurements show good agreement with FONLL predictions (empty/filled boxes). 


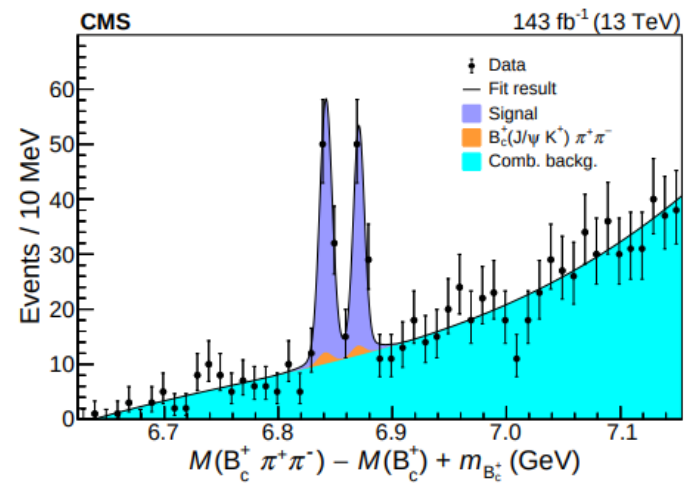

Figure 3: The invariant mass distribution for $B_{c}^{+} \pi^{+} \pi^{-}$candidates, with the mass value of the $B_{c}^{+}$candidate kinematically constrained to the nominal one. The UML fit result is superimposed. The $B_{c}^{*}(2 S)^{+}$and $B_{c}(2 S)^{+}$signals correspond respectively to the lower-mass and higher-mass peaks. The measured mass difference between the two signals is $\Delta \mathrm{M}=(28.9 \pm 1.5) \mathrm{MeV}$ [7].

\section{Measurement of $B_{c}(2 S)^{+}$and $B_{c}^{*}(2 S)^{+}$cross section ratios}

The CMS Collaboration recently reported the observation of two well-resolved peaks in the $B_{c}^{+} \pi^{+} \pi^{-}$invariant mass distribution, with $B_{c}^{+} \rightarrow \pi^{+} J / \psi\left(\rightarrow \mu^{+} \mu^{+}\right)$, using $143 \mathrm{fb}^{-1}$ of pp collisions data collected between 2015 and 2018 [6]. The $B_{c}^{+}$candidates are reconstructed in the $J / \psi \pi^{+}$decay channel. The two peaks in Fig. 3 have been identified as $B_{c}(2 S)^{+}$and $B_{c}^{*}(2 S)^{+}$signals and their signal yields are extracted with an UML fit. This analysis has been recently completed by measuring the $B_{c}(2 S)^{+}$to $B_{c}^{+}, B_{c}^{*}(2 S)^{+}$to $B_{c}^{+}$, and $B_{c}^{*}(2 S)^{+}$to $B_{c}(2 S)^{+}$cross section ratios (respectively $R^{+}$, $R^{*+}, R^{*+} / R^{+}$) [7]. The yields enter the ratios after they are corrected by the relative efficiencies, estimated with $\mathrm{MC}$ techniques. The kinematic range analyzed is defined by the $B_{c}^{+}$transverse momentum $p_{T}>15 \mathrm{GeV}$ and rapidity $|y|<2.4$. These ratios include the unknown branching fractions of the $B_{c}^{(*)}(2 S)^{+} \rightarrow B_{c}^{(*)+} \pi^{+} \pi^{-}$decays. The $B_{c}^{*+}$ meson is assumed to decay with a branching fraction of $100 \%$ in $B_{c}^{*+} \rightarrow B_{c}^{+} \gamma$, where the photon is not reconstructed ( $E_{\gamma} \simeq 55 \mathrm{MeV}$ ).

The three ratios as a function of $p_{T}\left(B_{c}\right)$ and $y\left(B_{c}\right)$ show no significant dependence on the two variables (left and middle plot in Fig. 4, respectively).
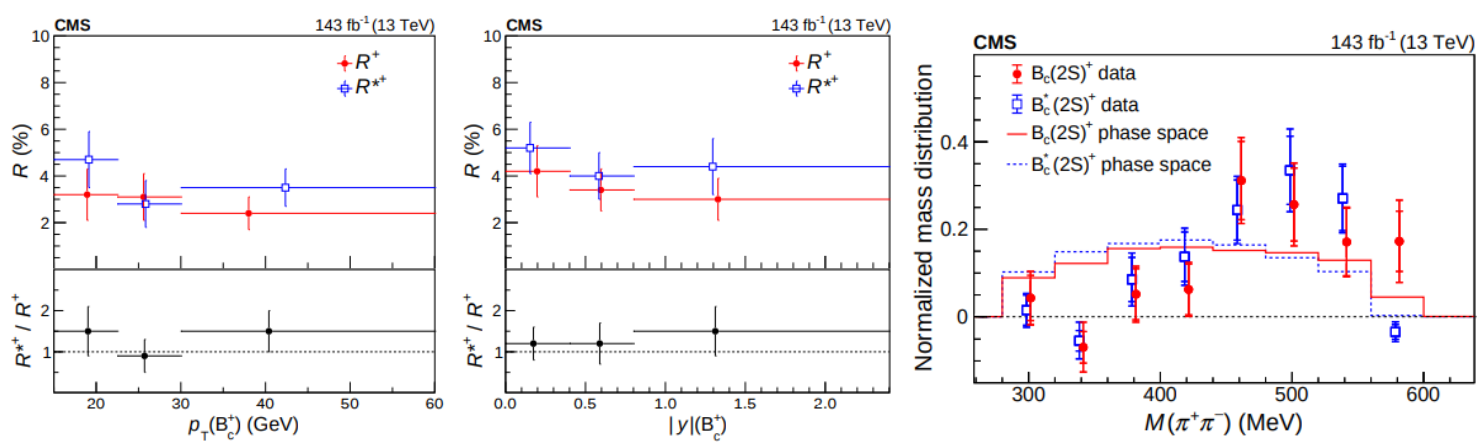

Figure 4: Differential cross section ratios for $R^{+}, R^{*+}, R^{*+} / R^{+}$with respect to transverse momentum (left) and rapidity (center) of the $B_{c}^{+}$meson. No dependence on these variables is observed. Invariant mass of the di-pion system (right). The distributions for $B_{c}(2 S)^{+}$and $B_{c}^{*}(2 S)^{+}$candidates are background-subtracted and compared with pure phase-space model. 
The normalized dipion invariant mass distributions for the two excited states have been also investigated (right plot in Fig. 4): different shapes with respect to the phase space profile are observed, but this difference is not fully significant at this level of statistics and uncertainties.

\section{Measurement of fiducial cross section for $\Upsilon(1 S)$ pair production}

The CMS Collaboration has measured the cross section for $\Upsilon(1 S)$ pair production in the fiducial region $|y(\Upsilon(1 S))|<2.0$ with $35.9 \mathrm{fb}^{-1}$ of pp collisions data collected in 2016. In addition, the different contributions to the production process from the two production mechanisms, singleparton scattering (SPS) and double-parton scattering (DPS), are measured for the first time [8]. Events are collected using a trigger which requires an opposite-sign muon pair compatible with $\Upsilon(1 S)$ and an additional muon coming from the same vertex. Further offline selection retains only events with four muons in the final state paired in two bottomonia.

Reconstruction efficiency and acceptance are estimated with simulation studies. Data are corrected event-by-event with efficiency weights, then the signal yield is extracted by performing a two-dimensional UML fit on the invariant masses of the two opposite-sign muon pairs (Fig. 5).

The result on the fiducial cross section is obtained assuming $\Upsilon(1 S)$ is produced unpolarized: $\sigma_{\text {fid }}=79 \pm 11$ (stat) $\pm 6($ syst $) \pm 3(\mathcal{B}) \mathrm{pb}$, where the last term corresponds to the uncertainty in the world-average branching ratio of the $\Upsilon(1 S)$ dimuon decay. The result is consistent with the previous measurement performed by CMS at $\sqrt{s}=8 \mathrm{TeV}$ [9].

Studies on simulation show that the most useful variable to distinguish between the SPS and DPS contributions is the angular separation between the two bottomonia $|\Delta y(\Upsilon(1 S), \Upsilon(1 S))|$. The distributions for two production mechanisms are separately extracted from MC generation, while the DPS-to-inclusive fraction $f_{D P S}$ is a free parameter in the fit of the aforementioned distribution (Fig. 6). The result obtained from the fit on $|\Delta y(\Upsilon(1 S), \Upsilon(1 S))|$ is $f_{D P S}=0.39 \pm 0.14$.
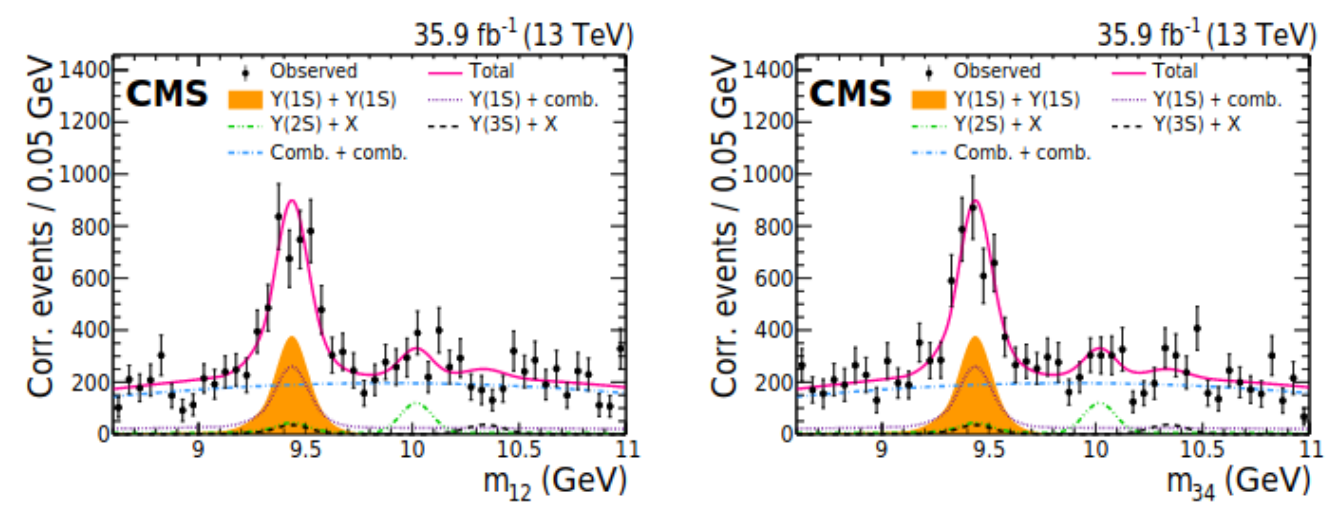

Figure 5: 2D UML fit on the muon pairs invariant masses distribution. Data (black dots) are corrected by efficiency and acceptance event-by-event. Each $\Upsilon(1 S)$ is modeled with the sum of two Crystal-Ball functions with common mean, in order to describe the detector non-uniform response. The contributions from $\Upsilon(2 S)$ and $\Upsilon(3 S)$, which are considered as background for this analysis, are modelled with gaussian functions. The combinatorial background is modelled with a $2^{\text {nd }}$-order Chebyshev polynomial. 


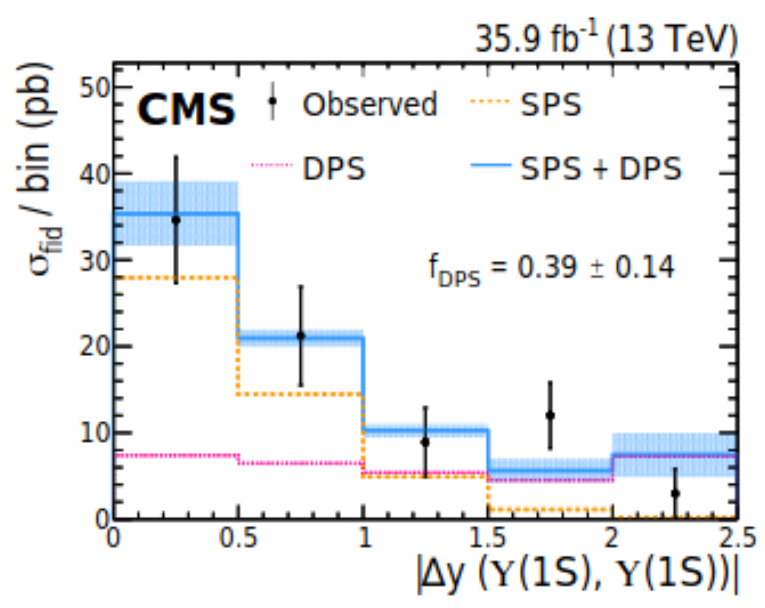

Figure 6: Black dots represent data, after efficiency and acceptance correction, for the fiducial $\Upsilon(1 S)$ pair production cross section in $|\Delta y(\Upsilon(1 S), \Upsilon(1 S))|$ bins. The shapes for DPS (pink dotted line) and SPS (yellow dotted line) contributions are extracted separately from MC studies.

\section{References}

[1] CMS Collaboration, The CMS experiment at the CERN LHC, JINST 3 S08004 (2008)

[2] CMS Collaboration, Measurement of prompt open-charm production cross sections in protonproton collisions at $\sqrt{s}=13 \mathrm{TeV}$, CMS-PAS-BPH-18-003 (2020)

[3] M. Cacciari, M. Greco, and P. Nason, The pT spectrum in heavy flavor hadroproduction, JHEP 05007 (1998)

[4] ATLAS Collaboration, Measurement of $D^{* \pm}, D^{ \pm}, D_{s}^{ \pm}$meson production cross sections in $p p$ collisions at $\sqrt{s}=7 \mathrm{TeV}$ with the ATLAS detector, Nucl. Phys. B. 907 717-763 (2016)

[5] LHCb Collaboration, Measurements of prompt charm production cross-sections in pp collisions at $\sqrt{s}=13 \mathrm{TeV}$, JHEP 201774 (2017)

[6] CMS Collaboration, Observation of two excited $B_{c}^{+}$states and measurement of the $B_{c}(2 S)^{+}$ mass in pp collisions at $\sqrt{s}=13 \mathrm{TeV}$, PRL 122132001 (2019)

[7] CMS Collaboration, Measurement of $B_{c}(2 S)^{+}$and $B_{c}^{*}(2 S)^{+}$cross section ratios in protonproton collisions at $\sqrt{s}=13 \mathrm{TeV}$, PRD 102092007 (2020)

[8] CMS Collaboration, Measurement of the $\Upsilon(1 S)$ pair production cross section and search for resonances decaying to $\Upsilon(1 S) \mu^{+} \mu^{-}$in proton-proton collisions at $\sqrt{s}=13 \mathrm{TeV}$, PLB 808 135578 (2020)

[9] CMS Collaboration, Observation of $\Upsilon(1 S)$ pair production in proton-proton collisions at $\sqrt{s}=8 \mathrm{TeV}$, JHEP 05013 (2017) 